HNO 2020 • 68:391-392

https://doi.org/10.1007/s00106-020-00833-0

(c) Springer Medizin Verlag $\mathrm{GmbH}$, ein Teil von Springer Nature 2020

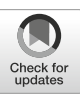

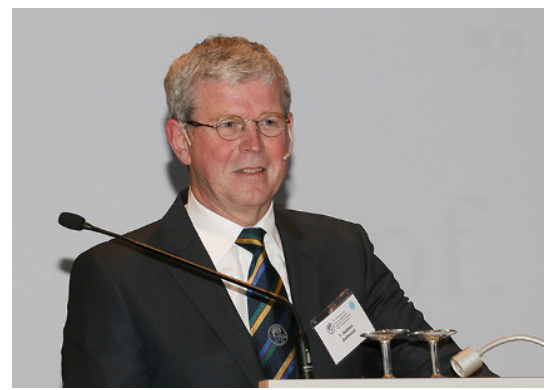

A HNO-Kongress 2014, Präsident T. Deitmer. Copyright DGHNO KHC

Etwa $30 \%$ aller Patienten in der HNOHeilkunde sind Kinder. Der Umgang mit und die Behandlung von Kindern ist daher Alltag für praktisch jeden klinisch tätigen HNO-Arzt.

\section{》) Die Behandlung von Kindern ist Alltag für praktisch jeden klinisch tätigen HNO-Arzt}

Die Struktur des Fachs spiegelt diese Tatsachen aber kaum wieder. So gibt es keine spezifische Weiterbildung oder formale Zusatzbezeichnung und auch keine eigene Fachgesellschaft für die doch in vielen Aspekten sehr unterschiedliche Situation dieser Altersgruppe. Im internationalen Vergleich bewegt sich Deutschland damit auf einem Sonderweg. In praktisch allen westlichen Industrieländern besteht eine Subspezialisierung mit z. T. eigener Facharztbezeichnung für die pädiatrische HNO-Heilkunde. Eigene Fachge-

\title{
C. Sittel
}

Klinik für Hals-, Nasen-, Ohrenkrankheiten, Plastische Operationen, Klinikum Stuttgart, Stuttgart, Deutschland

\section{Pädiatrische HNO-Heilkunde in Deutschland: Standortbestimmung und Ausblick}

\section{Festschrift für Thomas Deitmer}

sellschaften haben zur Bildung von Netzwerken auf lokaler und nationaler Ebene geführt, welche wiederum mit der European Society of Pediatric Otolaryngology (ESPO) ihre supranationale Entsprechung findet. Der alle 2 Jahre stattfindende Kongress der ESPO ist in der Folge zum weltgrößten Kongress für HNOHeilkunde und ihre Grenzgebiete im Kindesalter gereift und stellt eine einzigartige Wissensbörse und Vernetzungsoption bereit.

Mit der Gründung der Arbeitsgemeinschaft PädHNO soll an diese internationale Entwicklung angeknüpft werden. Die spezielle Kontur unserer Subspezialität soll geschärft und damit nach innen und außen besser sichtbar werden. Das Bewusstsein über die differenten Anforderungen im Kindesalter ist die Voraussetzung für die Anpassung entsprechender Strukturen und für die intensive Beschäftigung mit fortgeschrittenen Behandlungsmöglichkeiten. Davon profitieren alle Beteiligten, sowohl Patienten als auch Ärzte, die sich täglich mit kindlichen HNO-Problemen beschäftigen. Für spezielle Fragestellungen hilft die Bildung eines Netzwerks um sicherzustellen, dass seltene Fälle in ausreichend erfahrenen Zentren behandelt werden. Zweifellos wird in Deutschland schon heute in vielen Bereichen, etwa der Otologie und der Atemwegschirurgie, pädiatrische HNO-Heilkunde auf sehr hohem Niveau betrieben. Das gegenseitige Wissen um diese Zentren und deren Spektrum ist aber nicht transparent abrufbar und nicht allgemein zugänglich.

Thomas Deitmer, dem dieses Heft gewidmet ist, hat sich schon sehr lange um die Kinder in der HNO-Heilkunde in besonderer Weise bemüht und war die treibende Kraft hinter der Gründung einer eigenen Arbeitsgemeinschaft, deren Gründungsvorsitzender er war. Mit dem vorliegenden Heft soll sein vielfältiges Engagement geehrt werden und zugleich eine Standortbestimmung und Netzwerkbildung im oben ausgeführten Sinne vorangetrieben werden.

Die heterogene Situation in der Versorgung nach HNO-Eingriffen im Krankenhaus wird von Windfuhr et al. dargestellt. Für manche sicher überraschend ist der Befund, dass schon heute über die Hälfte aller Kinder nach HNO-Eingriffen in der primären Obhut von unseren pädiatrischen Kollegen betreut werden. Sofern die Organisationsstrukturen eine unmittelbare Eingriffsmöglichkeit des HNO-Facharztes im Fall von Komplikationen gewährleisten, erscheint dieser Trend im Interesse von Kindern und Eltern, da Aufenthalts- und Betreuungsqualität im pädiatrischen Umfeld sicher höher sind. Erfreulicherweise kann der Artikel zeigen, dass diese organisatorischen Voraussetzungen in allen Abteilungen, die an der Umfrage teilgenommen haben, in hohem Umfang gewährleistet sind. 
Die Übersicht von Grundmann zur chirurgischen Therapie komplexer Fehlbildungen des kindlichen Ohrs zeigt die Vielfalt des möglichen Behandlungsspektrums. Während das grundsätzliche Wissen über die therapeutischen Optionen möglichst breit gestreut werden sollte, ist die operative Therapie dieser seltenen Erkrankungen ein typisches Beispiel für die Sinnhaftigkeit überregionaler Zentrumsbildung.

\section{》) Über die Hälfte aller Kinder werden nach HNO-Eingriffen primär pädiatrisch betreut}

Sittel et al. propagieren die Verwendung der ELS-Klassifikation (endoskopische Diagnostik laryngotrachealer Stenosen) in der Diagnostik kindlicher Atemwegsstenosen. Mit diesem einfachen, auf dem bekannten Cotton-Myer-System aufbauenden Schema gelingt eine standardisierte und prognoserelevante endoskopische Diagnostik laryngotrachealer Stenosen im Kindesalter. Die qualitativ hochwertige und zuverlässige endoskopische Diagnostik sollte mehr noch als bisher flächendeckend zur Verfügung gestellt werden können. Sie ist die Grundlage für das Erkennen therapeutischer Notwendigkeiten und Möglichkeiten, die dann wiederum sinnvollerweise an ein erfahrenes Zentrum delegiert werden sollten.

Windfuhr et al. präsentieren in $2 \mathrm{Ar}$ beiten die statistischen Daten zur bundesweiten Tonsillenchirurgie. Sehr deutlich kann gezeigt werden, dass die Operationsfrequenz auch schon vor der aktuellen und viel diskutierten Neufassung der Leitlinie bundesweit deutlich gesunken ist. Wie so oft in der Medizin muss man sich auch hier die Frage stellen, ob mittlerweile das Pendel nicht allzu sehr zur Gegenrichtung ausgeschlagen hat. Die Indikation einer Tonsillektomie wird vielerorts außerordentlich streng gestellt, wobei in der Abwägung der Argumente die grundsätzlich unzweifelhafte Sinnhaftigkeit der Tonsillektomie aus der Perspektive zu geraten droht.

Die Arbeitsgemeinschaft PädHNO der DGHNO hofft auch, mit diesem
Heft das Interesse an der kindlichen HNO-Heilkunde wachzuhalten und zu vertiefen. Für die Zukunft hoffen wir auf viele pädiatrisch interessierte Kollegen, die das Profil und die Wirkung der Arbeitsgemeinschaft im Interesse unserer kleinen Patienten schärfen und verstärken.

\section{Sittel}

\section{Korrespondenzadresse}

Prof. Dr. med. C. Sittel
Klinik für Hals-, Nasen-,
Ohrenkrankheiten, Plastische
Operationen, Klinikum
Stuttgart
Kriegsbergstraße 60,
70174 Stuttgart, Deutschland
c.sittel@klinikum-stuttgart.de

Interessenkonflikt. C. Sittel gibt an, dass kein Interessenkonflikt besteht.
HNO-CORONA-NEWS-TICKER

https://www.hno.org/de/corona

Sehr geehrte Kolleginnen und Kollegen, die DGHNO-KHC hatte bekanntermaßen bislang auf ihrer Website hno.org im Zusammenhang mit der Corona-Krise auf die Verlautbarungen des Robert-KochInstitutes verlinkt. Dahinter steht die Erkenntnis, dass die Informationen von dort gesichert und geprüft sind, diese regelmäBig aktualisiert werden und die DGHNOKHC weder die Spezialisierung noch die Ressourcen hat, eine vergleichbar valide und anerkannte Information regelmäßig bereitzustellen.

Die Lage hat sich nunmehr dahingehend geändert, dass wissenschaftlich geprüfte Verlautbarungen bzw. Erfahrungen von internationalen HNO-Fachkolleg*innen bekannt werden, die Spezifika für unser HNO-Fachgebiet beschreiben. Im Fazit gibt es Erkenntnisse, dass HNO-Ärzt*innen neben Augen- und Zahnärzt*innen in ihrer Tätigkeit besonders gefährdet sind. Diese Erkenntnisse wollen wir Ihnen ab sofort über die Homepage der Deutschen HNO-Fachgesellschaft (DGHNO-KHC) in enger Abstimmung mit dem Deutschen HNO-Berufsverband (BVHNO) unter der Überschrift HNO-Corona-News-Ticker zur Verfügung stellen und unsere Empfehlungen regelmäßig anpassen.Die Informationen könnten für Sie zu Fragen des Arbeitsschutzes Ihrer Mitarbeiter*innen aber auch in Gesprächen mit Ihrer KlinikGeschäftsführung und Krankenhaushygiene wichtig sein.

Prof. Dr. med. Andreas Dietz, Leipzig Präsident DGHNO-KHC

Prof. Dr. med. Thomas Deitmer, Bonn Generalsekretär DGHNO-KHC

Dr. med. Dirk Heinrich, Berlin Präsident BVHNO

Hinweis: Teilen Sie uns Ihre klinischen Erfahrungen mit Corona-Patienten mit, die für unsere tägliche Arbeit wichtig sind. Bitte kontaktieren Sie dazu die folgende, speziell dafür eingerichtete E-MailAdresse corona@hno.org Quelle:

https://www.hno.org/de/corona 